\title{
Skills Matter: Modeling the relationship between decision making processes and collaborative problem-solving skills during Hidden Profile Tasks
}

\author{
Yiwen Lin \\ yiwenl21@uci.edu \\ University of California, Irvine \\ Irvine, California, USA
}

\author{
Andrew Godfrey \\ andgodfr@umich.edu \\ University of Michigan-Ann Arbor \\ Ann Arbor, Michigan, USA
}

\author{
Nia Dowell \\ dowelln@uci.edu \\ University of California, Irvine \\ Irvine, California, USA
}

\begin{abstract}
Collaborative problem-solving (CPS) is one of the most essential 21st century skills for success across educational and professional settings. The hidden-profile paradigm is one of the most prominent avenues of studying group decision making and underlying issues in information sharing. Previous research on the hidden-profile paradigm has primarily focused on static constructs (e.g., group size, group expertise), or on the information itself (whether certain pieces of information is being shared). In the current study, we propose a lens on individual and group's collaborative problem-solving skills, to explore the relationships between dynamic discourse processes and decision making in a distributed information environment. Specifically, we sought to examine CPS skills in association with decision change and productive decision-making. Our results suggest that while sharing information has significantly positive association with decision change and effective decision-making, other aspects of social processes appear to be negatively correlated with these outcomes. Cognitive CPS skills, however, exhibit a strong positive relationship with making a (productive) change in students final decisions. We also find that these results are more pronounced at the group level, particularly with cognitive CPS skills. Our study shed lights on a more nuanced picture of how social and cognitive CPS interactions are related to effective information sharing and decision making in collaborative problem-solving interactions.
\end{abstract}

\section{CCS CONCEPTS}

- Human-centered computing $\rightarrow$ Computer supported cooperative work.

\section{KEYWORDS}

collaborative problem solving, Hidden-profile paradigm, decision making, group processes

\section{ACM Reference Format:}

Yiwen Lin, Andrew Godfrey, and Nia Dowell. 2021. Skills Matter: Modeling the relationship between decision making processes and collaborative problem-solving skills during Hidden Profile Tasks. In LAK21: 11th International Learning Analytics and Knowledge Conference (LAK21), April

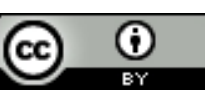

This work is licensed under a Creative Commons Attribution International 4.0 License.

LAK21, April 12-16, 2021, Irvine, CA, USA

(C) 2021 Copyright held by the owner/author(s)

ACM ISBN 978-1-4503-8935-8/21/04.

https://doi.org/10.1145/3448139.3448180
12-16, 2021, Irvine, CA, USA. ACM, New York, NY, USA, 10 pages. https: //doi.org/10.1145/3448139.3448180

\section{INTRODUCTION}

Key Competencies[36] or 21st Century Skills[18, 29] have been identified as critical across disciplines. Collaborative problem-solving (CPS) skill is one of the most essential of these 21st century skills that students must master in order to be successful in life [5, 13, 15, 17, 50]. Many problems in the modern world across home, workforce, and community spaces require teams to integrate group achievements with team members' idiosyncratic knowledge[17]. In the context of collaborative learning, the ability for individuals and teams to communicate ideas and achieve a collective decision after discussion is fundamental to effective problem-solving. Given the dynamic and complex nature of collaborative interactions, it is necessary to further dissect how specific CPS skills may benefit or hinder collaborative outcomes. Increasing, learning analytics research calls for the development of salable assessments with regards to the social and cognitive domains of collaborative problem-solving $[6,10,14]$, and emphasizes the need to expand the task scenario of CPS studies to inform more naturalistic problemsolving situations[16].

Our current research investigates the relationship between individual and team CPS skills and decision-making outcomes through collaborative discourse in a hidden-profile task[42, 43]. We combine conceptual insights from the fields of social psychology, learning sciences, and methods of learning analytics to propose a novel perspective in gaining understanding towards collaborative interactions. Specifically, we adopt a CPS assessment framework theoreticallygrounded in learning sciences to examine learners interactions across social and cognitive dimensions in a collaborative decision making environment.

An other unique aspect of our study is the employment of the hidden profile scenario. A hidden profile paradigm, which we explain in greater details in the subsequent section, is an experimental set up to simulate real world problem-solving situation where knowledge is distributed, and learners do not have identical information relevant to the problem they need to solve[44]. It also offers a proxy to collaborative learning in general, where learners with different backgrounds skills socially negotiate and construct shared knowledge in the process of finding solution to the problem[35]. Successfully navigating the scenario require CPS members to exchange information and organize teamwork (i.e. planning and monitoring), highlighting communication and facilitation skills that are fundamental to all collaborative learning settings. While hidden 
profile research in social psychology traditionally have a focus on understanding the effect of static constructs (e.g., group size, group composition, task characteristics, information technology), we took a learner-centered and process-oriented perspective from a learning analytics standpoint. Towards this effort, we use content analysis to quantify CPS skills emerged in CPS discourse and modeled its relationship with information sharing and decision-making outcomes. Additionally, we examined the influence of CPS skills at both individual and group levels. This multilevel analysis allows us to observe how strongly CPS skills and processes are relevant to CPS outcome for each individual team members versus the team as a whole.

In the following sections, we provide an overview of existing literature around group decision making and a description of the hiddenprofile paradigm. We then discuss the importance of learner's CPS skills and sociocognitive engagement to collaborative decision making, particularly in the context of a hidden-profile task. We move on to our methodological details including our experiment setup, measures of decision-making outcomes, as well as the CPS framework we use to quantify social and cognitive skills from collaborative discourse. We present our statistical analysis using linear mixedeffects models before moving onto discussing our findings and implications for future CPS research.

\section{RELATED WORK}

\subsection{Hidden Profile Task-Collaborative Information Sharing}

The hidden-profile paradigm is one of the most fruitful avenues of research on group decision making and information sharing today. In a typical hidden-profile task, information is unevenly distributed among group members prior to group discussion, whereby some is shared by all group members and some is unique to individual members[42]. In other words, each group member possesses information that the other group members do not have. The key to successfully solve the paradigm is to integrate this unshared information with the common information that every group member has. By integrating shared and unshared information, groups have a larger chance to arrive at a different, better decision alternative than each member's information alone[46]. A major challenge in this paradigm is to overcome initial biases based on incomplete information in order to see beyond the suboptimal choice. The key to successfully solving this paradigm requires each team member to share unique information, and effective teamwork as a whole to collectively come to the optimal solution[37].

Previous research using a hidden-profile paradigm reveals several key factors that affect information sharing and processing. For instance, studies have investigated the effects of group size[45], group composition[4, 19, 27], task features (e.g., task type, task demonstrability, hidden profile strength), and communication medium [34, 48, 49]. Studies also concerns information pooling: what information is being shared, how it is being discussed, and how it influences group decisions[33].

In our review of the literature, we found that research efforts have primarily been directed towards team-level attributes, while focuses on individual level attributes have been relatively scarce. Among a couple studies explored individual competency in relation to information sharing and seeking, competency has been referred to as task-related expertise, domain knowledge, or perception of relative competence[7]. To our knowledge, no study has yet explored communication and CPS skills as key competencies in association to hidden-profile task performance.

From the CPS perspective, the ability of an individual to deliver and process information, and engage in social regulatory activities in groups (e.g., planning and monitoring) is critical to successful collaborations. Social regulatory skills may play an even more important role in collaborative decision making contexts, such as hidden-profile tasks, as these skills would determine how team members share and navigate information to arrive at a judgment. In particular, in hidden-profile tasks, team members have different information, so they need to explicitly ask questions and volunteer information through acts of communication in order to facilitate team members having a common understanding[12, 17]. From this perspective, CPS skills can be considered an important individual attribute, or "member-level input" factor that influences decision making outcomes as these skills can be modeled to take into account the interplay of individual characteristics and group outcomes. Thus, it is important to recognize that team outcomes are fundamentally driven by individuals' ability to engage in group discourse and contribute to effective team decisions[6, 11, 38, 47]. In the current research, we draw on both the CPS and group decision making literature (i.e., input-output model) to provide a unique investigation into how these factors influence individual student and team decision making during a hidden profile interaction. In doing so, we are able to conceptualize students' CPS skills as individual level attributes or "member-level input" factors and assess their relationship to decision-making outcomes.

\subsection{CPS and Decision Making}

Decision making is a complex cognitive process where an individual or group gathers and organizes information, identifies and assesses alternative resolutions to form opinions and make choices. Within the context of CPS, decision making can be designed as an individual or group task, distinguished by whether individuals make separate decisions independently or come to one conclusion jointly with others after a group discussion on the information. Historically, studies suggest strong links between CPS processes and collective decisions made by groups[22]. However, the relationship between individual level processes (i.e., independent decision making) and group CPS behavior has not been thoroughly explored. In this section, we turn to past literature to identify what corresponding group processes may be related to decision-making outcomes. Our primary interest in doing so is to establish a connection between individual decisions as a CPS outcome and CPS processes captured by assessment framework.

34, Research has a long standing interest in individual decision making behavior in groups. Opinion shift, or decision change before and after group discussion, is often considered an indicator of group influence on individuals. A series of experiments from past to present provide detailed accounts for how and why opinion shifts occur. Earlier studies in social psychology found social conformity in groups pressures individuals to change their answers to align with the group majority opinion[2][3]. Although when individuals are 
allowed to submit their choices privately, they are more likely to hold onto their initial positions. This assumption was proven to be true in digital platforms as well[40]. These evidence suggests that social conformity might be less likely to be the reason why individuals change their decisions in digitally-mediated groups. In the context of hidden-profile, a literature review shows that socialpsychological factors mediate decision-making outcomes by having an influence on communication processes[41]. This prompted us to consider an alternative construct that individual decision change more accurately represents in digitally-mediated CPS.

A replication of Asch's study found that new information plays an important role in individual opinion change[26]. This suggests that opinion change signals the impact of new information in individual decision making. In the hidden profile situation that we describe further below, presenting new and unique information is key to solving the problem. Therefore, we propose that opinion shifts between pre- and post group discussion could be seen as an indicator of the effect of information sharing on decision-making. To elaborate further, we perceive the discrepancy in two occurrences of decisions reflect social processes where individuals or groups share information and exchange opinions. Thereby through examining the relationship between CPS skills and decision change, we intend to explore how CPS skills predict information sharing behavior.

However, sharing information alone is not equivalent to successful problem-solving. The challenge of solving the hidden-profile task is that individuals must re-evaluate the situation and they must change their answers in order to reach the optimal choice. Therefore, we need a second construct to indicate whether a team member effectively processes shared information in a group. This can be measured by further looking at whether a decision change leads to an accurate decision. By examining the relationship between CPS skills and productive decision change, we intend to capture how CPS skills predict effective information processing.

\subsection{CPS Skills Assessment}

Researchers previously expressed the need for more in-depth analysis around the role of social and cognitive mechanisms in information sharing and decision-making processes[41]. To complement the lack of sociocognitive focus in hidden-profile research, we found multiple small group collaboration studies highlighting the importance of considering social and cognitive dynamics of group communication through collaborative discourse[9, 11, 12]. However, CPS skills are traditionally difficult to capture given the diverse sets of skills within the social and cognitive dimensions [1]. There are different existing CPS assessment frameworks that were developed to capture the complexity of human collaboration. A widely adopted framework developed by the Programme for International Student Achievement (PISA) employed the human-to-agent mode for the CPS assessment [29]. This framework identifies three social competencies, including establishing and maintaining shared understanding, taking appropriate action to solve the problem, and establishing and maintaining team organization. Additionally, the framework also conceptualizes four problem solving or cognitive processes, including exploring and understanding, representing and formulating, planning and executing, and monitoring and reflecting. Educational Testing Service (ETS) has an alternative framework with an emphasis on integrating functions of both social and cognitive dimensions of CPS. This CPS framework includes four broad CPS skills: sharing ideas, assimilating and accommodating knowledge/perspective taking, regulating problem solving ideas, and maintaining positive communication[25]. Some studies modify and adapt constructs from these frameworks to tailor to measuring CPS in a particular knowledge domain[20]. Others expand CPS assessment into an ontology that establishes theory-driven connection between skills and behavior. In order to conceptualize the CPS construct most suitable to our hidden-profile study, we adapted our assessment rubric from Andrews-Todd \& Forsyth's [1] CPS framework, which we further describe in the method section.

\section{OVERVIEW OF PRESENT STUDY}

The goal of this research is to zoom in on the CPS skills that diagnostically reveal decision making outcomes across levels of multi-party ecologies. We considered two possible channels that CPS skills may be related decision-making outcomes. In our first research question, by asking how CPS skills captured from group discourse are associated with the degree to which individuals in the group change their opinion from pre-discussion preferences, we are interested in the effect of group information sharing, regardless of whether they are changing in the correct direction or not. The second part of the question asks how individual's CPS skills exhibited at the group discussion predict how they make decisions later. We assume that an individual's propensity to share information and engage in group processes may be indicative of their ability in gathering useful information from group discussion to form optimal decisions.

Our second research question is built upon the assumption that CPS skills aggregated at the group level may suggest information exchange dynamics and effectiveness. We hypothesize stronger relationships between collective CPS skills of a group and the proportionated decision making outcomes. We also hypothesize that social and cognitive CPS skills might be meaningfully related to collaboration outcomes differently. In this regard, we expect that some CPS skills regarding the group structuring and functioning might be more relevant to group collaboration outcomes than for each individual.

For both individual and groups alike, information sharing is key to delivering hidden information, which is a premise for making effective decisions. However, merely presenting facts about profiles may not lead to optimal decisions. In the group setting, productive teamwork might rely more on cognitive discourse in setting shared goals, proposing strategic plans for problem-solving, and communicating team member's actions in order to process new information effectively. As such, we seek to understand what aspects of social and cognitive skills respectively influence individual and group outcomes.

To summarize, our research questions are:

- RQ1: How do individual CPS skills relate to influence: opinion shift (from pre-discussion preferences) and whether the change is productive (i.e.achieved the optimal solution)?

- RQ2: How do CPS skills exhibited at the group level influence: the degree to which opinion shifts happen in a group, and the 
degree to which team members make a productive decision change?

\section{METHODS}

\subsection{Participants}

A total of $\mathrm{N}=525$ undergraduate students from a large university in the U.S. southwest participated in the study. Participants were randomly assigned into teams to take part in a Hidden Profile CPS task. In total $\mathrm{N}=134$ teams were included in the study. Teams are predominantly four person groups with a few exceptions that consist of three or five people. Over a half of our participants were female $(\mathrm{N}=363)$, and most of them were freshman $(\mathrm{N}=354)$ or junior $(\mathrm{N}=137)$. Of those participants who reported their race and ethnicity (508 out of 525), $12.8 \%$ of the participants were White, $1.8 \%$ were Black or African American, $44.1 \%$ were Asian, $32.7 \%$ were Hispanic or Latino, and $2 \%$ were multiracial.

\subsection{Procedure}

The ETS Platform for Collaborative Assessment and Learning (EPCAL) was used as a platform for this study. The ETS Collaborative Science Assessment Prototype (ECSAP) was used to measure learners CPS skills, outcomes [20]. Prior to completing the CPS task, participants individually completed a background questionnaire that included items to obtain information such as participants' race, gender, age, highest level of education completed, native language, computer use, and parents' highest level of education. Participants were then asked to complete a collaborative problem-solving task that involved decision-making using an online platform. The hidden profile paradigm as described above distributes common information among team members and reserves unique information to each participant. In our study, participants were presented a set of features about three options in one of four task conditions (i.e., apartments, professors, party venue, and job candidates). Some of the features were known to all participants prior to group discussion, while others were unshared features (i.e., only one student received that feature information).

The study consisted of two parts. In the first part, participants were randomly assigned a task condition and were given $10 \mathrm{~min}$ utes to study the material. The reading material presented three choices with facts marked by positive, neutral, or negative points. Participants were asked to evaluate each choice and rank each one from the best to the worst. For instance, in the candidate scenario, participants were asked to choose a person for promotion at a workplace. All participants saw several shared traits about candidate $C$, such as "is able to offer criticism in a supportive way (+)" and "lack of organization (-)". Only one participant saw candidate C was also "warm, friendly, and outgoing (+)", and "swam on the varsity team in college (0)"; another participant saw information about candidate C that included "collaborates effectively with colleagues $(+)$ " and "enjoy listening to audio book (0)". We manipulated the choices such that based on the information that participants had in this round, there was an optimal rank order. After participants submitted their individual answers, they were prompted to the second part of the task.

The second component of the task was a collaborative discussion. Participants were randomly assigned to small groups to discuss the options for 20 minutes. In the previous round, participants were informed which information was exclusive to them and which was available to all members of the group. The goal for participants in this round was to discuss features about the choices with their teammates and identify the optimal rank order. We manipulated the information so that successful completion of the task required groups to share unique information. In other words, to arrive at the accurate solution, participants would need the additional information from their teammates to make the best judgment. After the discussion, participants were asked to rank the options again based on the information they gathered after the group discussion and submit their decision independently. Participants were not required to submit a group answer after the discussion.

\subsection{Quantifying Hidden Profile CPS Task Outcomes}

Participants submitted both an individual answer, and a revised individual answer after team discussion. Students' responses to the seven multiple-choice equivalent items were scored based on the corresponding scoring rubrics as presented in[51]. In this way, the responses before collaboration capture each individual member's inquiry skills specific to the task, while the changes in individual student responses after the collaboration and the team responses reflect how effective the collaboration was and allow us to directly investigate which sociocognitive interaction dynamics may be more important for better collaboration skills and outcomes. Although these variables are correlated, they still provide a measure of how the interaction influences individual and team outcomes. Thus, in the current research we focus on the total correct score for individual student revised responses and the team response to quantify individual student and team level outcomes.

Specifically, we operationalized the hidden-profile CPS outcomes into two binary variables: decision change and productive decision change at both the individual and group level. At the individual level, decision change is computed by comparing whether participants' pre-discussion preference was consistent with their post-discussion answer. This measures the direct influence of collaborative discussion regardless of the accuracy of the decision. Secondly, we calculated the score change by subtracting the score participants received from revised answer and initial answer. Participants received two points if they got the fully correct order, and one point if they submitted a partially correct answer. We then generated the productive decision change based on whether or not individuals had a positive score change. This measure marks an improved individual decision after group discussion. For instance, if a participant submitted an incorrect answer prior to group discussion but received two points for submitting the accurate answer post discussion, it would be considered a productive decision change. Since the correct answer in the first and second part of the study were not the same, participants who made optimal decisions in both cases would have also changed their answers. However, this measure emphasizes whether the decision change improves individual performance rather than whether the decision change leads to an optimal outcome after group collaboration. In doing so, we narrow our focus on the effectiveness of collaborative discourse and reduce the variances might be explained by individual inquiry skills. At 
the group level, decision change is the proportion of individuals in the group who changed decisions pre- and post- discussion. This captures the extent to which group discussion influences an individual's opinion. Similarly, productive decision change is calculated as the proportion of individuals who had a positive score change in their second decision.

\subsection{Quantifying CPS Skills}

In addition to scoring the outcome responses, we also annotated the chat communication during the collaboration based on a slightly adapted version of Andrews-Todd \& Forsyth's [1]CPS framework. In particular, we removed one category that pertained to exploring the interactive features of the CPS environment, as this was not relevant for the current hidden-profile task. The adapted ontology included 8 high-level CPS skills across social and cognitive dimensions and 29 subskills that correspond to each high-level CPS skill. In this study, we exclusively focused on the 8 high level CPS skills across social and cognitive dimensions. The social dimension included four CPS skills, namely, maintaining communication (SMC), sharing information (SSI), establishing shared understanding (SESU), and negotiating (SN). The cognitive dimension included four CPS skills, namely, representing and formulating (CRF), planning $(\mathrm{CP})$, executing $(\mathrm{CE})$, and monitoring $(\mathrm{CM})$. A description of the 8 high-level CPS skills is provided in Table 1.

Coding was completed at the level of each log file event (i.e., chat submission) and each event could only receive one code. That is, the unit of analysis was each turn of a conversation, or each conversational utterance. A total of four raters coded the content of students' discourse recorded in the log data for the presence of 8 CPS skills. After having raters familiarized with the CPS framework and codebook, we asked raters to code the same random sample of $20 \%$ of all utterances in the data independently. This was repeated several times to make sure all discrepancies among raters were addressed and consensus was reached. The resulting average interrater reliability between all raters was found to be Kappa $=.81$, which is considered an acceptable inter-rater reliability (Kappa $>.60 ;[23])$. Then, the remaining data were evenly split into four groups and each group was coded independently by one of the four trained raters. There were a total of 7,711 utterance events that were coded. After coding the data, we were then able to inspect the frequencies of each of the 8 CPS skills. Specifically, for each student we calculated the proportional occurrence for each of the 8 categories of CPS skills (e.g., Sharing Information, Negotiating, Planning etc.). Additionally, at the team level we summed the total frequencies of CPS skills in the group dialogue, then calculated the proportional occurrence for each of the 8 categories.

\subsection{Statistical Analysis}

For all research questions, a mixed-effects modeling methodology was adopted[31]. Mixed-effects models include a combination of fixed and random effects, and can be used to assess the influence of the fixed effects on dependent variables after accounting for the random effects. Multilevel modelling handles the hierarchical nesting, interdependency, and unit of analysis problems that are inherent in collaborative learning data. They are the most appropriate technique for investigating data derived from group interactions [8, 21].
In addition to constructing the fixed effects models, null models with the random effects (the task: candidate, apartment, professor, and party venue) but no fixed effects were constructed. A comparison of the null random-effects only model with the fixed-effect models allows us to determine whether communication patterns predict student and team decision making above and beyond the variance attributed to task type. The Akaike Information Criterion (AIC), Log Likelihood (LL), and a likelihood ratio test were all used to evaluate the overall fit of the models. Additionally, the effect sizes for each model were estimated using a pseudo R2 method, as suggested by Nakagawa and Schielzeth[28]. For mixed-effects models, $R^{2}$ can be divided into two parts: marginal $\left(R_{m}^{2}\right)$ and conditional $\left(R_{c}^{2}\right)$. Marginal $R^{2}$ is associated with variance explained by fixed factors, whereas conditional $R^{2}$ can be interpreted as the variance explained by the entire model, namely random and fixed factors. Both the marginal and conditional parts convey unique and relevant information regarding the model fit and variance explained. The nlme package in $\mathrm{R}$ [32] was used to perform all the required computations.

\section{RESULTS}

For the first research question, in order to evaluate the effect of CPS skills on individual CPS outcomes purely at the individual level, linear mixed-effects models were constructed separately for social and cognitive CPS dimensions. We constructed: (i) two individual decision change models models for student Decision Change (i.e., Individual Social Process Decision Change model and Individual Cognitive Process Decision Change model regardless of correctness) as the dependent variable and. One model with four social CPS skills as independent variable and the other model with cognitive CPS skills as independent variables. (ii) two individual productive decision change models (i.e., Individual Social Process Productive Decision Change model and Individual Cognitive Process Productive Decision Change model) models for student Productive Decision Change (improved CPS performance) as the dependent variable. Null models contained only random effect, task, and no fixed effects were constructed. In addition, a null model with task scenario as random effects only and no fixed effects was constructed for comparison. All full models were compared with the null model to assess the prediction

The likelihood ratio tests indicated that the student Decision Change model for social dimension CPS skills has a result of $\chi^{2}(4)$ $=10.677, \rho<.01, R_{m}^{2}=.02, R_{c}^{2}=.29$ and yielded a significantly better fit than the null model. The likelihood ratio tests indicated that the student Decision Change model for cognitive dimension CPS skills has a result of $\chi^{2}(4)=10.124, \rho<.01, R_{m}^{2}=.019, R_{c}^{2}=.032$, also yielded a significantly better fit than the null model with only random effects. We performed the analysis separately for social and cognitive dimensions given that combining them in one single model would result in a saturated model. Similarly, for student productive student decision change model, the model comparison for social dimension yielded a significant fit, $\chi^{2}(4)=24.67, \rho<.001$, $R_{m}^{2}=.04, R_{c}^{2}=.16$. The cognitive dimensions model did not differ significantly from the null model, $\chi^{2}(4)=7.51, \rho>1, R_{m}^{2}=.01, R_{c}^{2}=$ .13 . 
Table 1: CPS skills description

\begin{tabular}{|c|c|c|c|}
\hline Dimension & Skills & Definition & Example \\
\hline \multirow[t]{4}{*}{ Social } & $\begin{array}{l}\text { Maintaining } \\
\text { Communication } \\
\text { (SMC) }\end{array}$ & $\begin{array}{l}\text { Building rapport, or off-topic } \\
\text { and inappropriate communication. }\end{array}$ & $\begin{array}{l}\text { "nice job guys" } \\
\text { "should have drank coffee } \\
\text { this morning" }\end{array}$ \\
\hline & $\begin{array}{l}\text { Sharing } \\
\text { Information } \\
\text { (SSI) }\end{array}$ & $\begin{array}{l}\text { Share positive and negative features in } \\
\text { the hidden profile, task relevant information, or } \\
\text { make statement indicating state of own understanding. }\end{array}$ & $\begin{array}{l}\text { "candidate A was listed as } \\
\text { having good leadership } \\
\text { skills" } \\
\text { "I don't get it" }\end{array}$ \\
\hline & $\begin{array}{l}\text { Establish } \\
\text { Shared } \\
\text { Understanding } \\
\text { (SESU) }\end{array}$ & $\begin{array}{l}\text { Request and responses for direct information } \\
\text { from teammates to ensure understanding. }\end{array}$ & $\begin{array}{l}\text { "Hey so what was } \\
\text { everyone’s ranking?" } \\
\text { "that's what I got" }\end{array}$ \\
\hline & $\begin{array}{l}\text { Negotiating } \\
(\mathrm{SN})\end{array}$ & $\begin{array}{l}\text { Express agreement or disagreement, express } \\
\text { quitting or conflict resolution. }\end{array}$ & $\begin{array}{l}\text { "yes, I agree" } \\
\text { "That’s not right" }\end{array}$ \\
\hline \multirow[t]{4}{*}{ Cognitive } & $\begin{array}{l}\text { Representing and } \\
\text { Formulating (CRF) }\end{array}$ & $\begin{array}{l}\text { Represent the problem or develop hypothesis } \\
\text { for how changes will affect the situations and } \\
\text { propose justifications. }\end{array}$ & $\begin{array}{l}\text { "Yeah I feel that B is the } \\
\text { best because everything is } \\
\text { nearby and the landlord } \\
\text { offers a } 24 \text {-hour maintenance service" }\end{array}$ \\
\hline & $\begin{array}{l}\text { Planning } \\
\text { (CP) }\end{array}$ & $\begin{array}{l}\text { Clarify the overall goal or propose change to } \\
\text { a different plan to solve the problem. }\end{array}$ & $\begin{array}{l}\text { "maybe should do it this } \\
\text { instead" }\end{array}$ \\
\hline & $\begin{array}{l}\text { Executing } \\
(\mathrm{CE})\end{array}$ & $\begin{array}{l}\text { Make suggestion for action for teammate to } \\
\text { carry out or communicate own actions. }\end{array}$ & $\begin{array}{l}\text { "I’ll look at my info for A } \\
\text { and C again real quick" }\end{array}$ \\
\hline & $\begin{array}{l}\text { Monitoring } \\
(\mathrm{CM})\end{array}$ & $\begin{array}{l}\text { Monitor progress toward the goal, including } \\
\text { checking intermediate and final results, or } \\
\text { monitor teammates are present. }\end{array}$ & $\begin{array}{l}\text { "You forgot to mention } \\
\text { one of the features" } \\
\text { "Let's get a move on } \\
\text { (name)" }\end{array}$ \\
\hline
\end{tabular}

Overall, as shown in Table 2, we observed that at the individual level, decision change has a significant negative relationship with Maintaining Communication (social), and a significant positive relationship with Execution (cognitive). From the results in Table 3 , we can see that only Information Sharing (social) was found to positively contribute to productive decision change. Cognitive CPS skills did not show significant correlation with productive decision change, with the exception of a marginally significant positive correlation between Execution and outcome $(p=.069)$.

Our second set of research questions focused on assessing the relationship between team level CPS skills and decision change (regardless of if it was optimal) and productive decision change. As such, followed a similar procedure as described for the individual level analyses. That is, we constructed two sets of models, i) two team decision change models (i.e., Team Social Process Decision Change model and Team Cognitive Process Decision Change model), and ii) two team productive decision change models (i.e., Team Social Process Productive Decision Change model and Team Cognitive Process Productive Decision Change model). Additionally, null models were only random effect, task, and no fixed effects were constructed. The likelihood ratio tests indicated that the Team Social Process Decision Change model with $\chi^{2}(4)=33.82, p<.001$, $R_{m}^{2}=.09, R_{c}^{2}=.12$ and the Team Cognitive Process Decision Change model $\chi^{2}(4)=41.57, p<.001, R_{m}^{2}=.15, R_{c}^{2}=.21$ both yielded a significantly better fit than the null model. For productive decision change outcome, the likelihood ratio tests indicated that the group
Productive Decision Change model for social dimension CPS skills has a result of $\chi^{2}(4)=22.87, p<.001, R_{m}^{2}=.13, R_{c}^{2}=.28$ and yielded a significantly better fit than the null models. The likelihood ratio tests indicated that the group Productive Decision Change model for cognitive dimension CPS skills has a result of $\chi^{2}(4)=41.57, p$ $<.001, R_{m}^{2}=.11, R_{c}^{2}=.28$ and also yielded significantly better fits than the null models.

Overall, as shown in Table 4, we observed that at the group level, decision change had a significant negative relationship with Establishing Shared Understanding (social), Maintaining Communication (social), and Negotiating (social) and a significant positive relationship with Planning (cognitive). As shown in Table 5, productive decision change at the group level had a significant negative relationship with Monitoring (cognitive) and significant positive relationships with Sharing Information (social), Executing (cognitive), and Planning (cognitive).

\section{DISCUSSION}

The current research presents a hidden-profile study to explore the relationship between CPS skills and collaborative decision-making outcomes. Our primary objective is to investigate how social and cognitive processes quantified by CPS skills assessment framework are related to information sharing and processing. A number of conclusions can be drawn from our statistics results. We discuss them further in the current section. 
Table 2: CPS skills and decision change (individual level)

\begin{tabular}{lccllcc}
\hline & \multicolumn{3}{c}{ Decision Change } & & \multicolumn{2}{c}{ Decision Change } \\
CPS Skills (Social) & $\beta$ & $C I$ & CPS Skills (Cognitive) & $\beta$ & $C I$ \\
\hline $\begin{array}{l}\text { Establish } \\
\text { Shared Understanding }\end{array}$ & -0.16 & $-0.59-0.27$ & $\begin{array}{l}\text { Representing } \\
\text { and Formulating }\end{array}$ & 0.20 & $-0.30-0.70$ \\
Maintaining & & & & & \\
Communication & $-0.53^{*}$ & $-0.95-0.12$ & Executing & $0.67^{*}$ & $-0.08-1.26$ \\
$\begin{array}{l}\text { Sharing Information } \\
\text { Negotiating }\end{array}$ & -0.05 & $-0.42-0.32$ & Monitoring & -0.23 & $-0.78-0.32$ \\
\hline Note: ${ }^{*} p<.05 ;{ }^{* *} p<.01 ;{ }^{* * *} p<.001 ;$ Estimates $\beta ; N=525$ & $-0.72-0.11$ & Planning & 2.62 & $-0.31-5.55$ \\
\hline
\end{tabular}

Table 3: CPS skills and productive decision change (individual level)

\begin{tabular}{lcclcc}
\hline & \multicolumn{2}{c}{ Productive Decision Change } & \multicolumn{2}{c}{ Productive Decision Change } \\
CPS Skills (Social) & $\beta$ & $C I$ & CPS Skills (Cognitive) & $\beta$ & CI \\
\hline $\begin{array}{l}\text { Establish } \\
\text { Shared Understanding }\end{array}$ & -0.19 & $-0.50-0.13$ & $\begin{array}{l}\text { Representing } \\
\text { and Formulating }\end{array}$ & -0.23 & $-0.60-0.15$ \\
$\begin{array}{l}\text { Maintaining } \\
\text { Communication }\end{array}$ & -0.11 & $-0.41-0.19$ & Executing & 0.41 & $-0.03-0.84$ \\
$\begin{array}{l}\text { Sharing Information } \\
\text { Negotiating }\end{array}$ & $0.32^{*}$ & $0.05-0.59$ & Monitoring & -0.23 & $-0.63-0.18$ \\
\hline
\end{tabular}

Note: ${ }^{*} p<.05 ;{ }^{* *} p<.01 ;{ }^{* * *} p<.001$; Estimates $\beta ; N=525$

Table 4: CPS skills and decision change (group level)

\begin{tabular}{lcclcc}
\hline & \multicolumn{2}{c}{ Decision Change } & & \multicolumn{2}{c}{ Decision Change } \\
CPS Skills (Social) & $\beta$ & \multicolumn{1}{c}{ CI } & CPS Skills (Cognitive) & $\beta$ & $C I$ \\
\hline $\begin{array}{l}\text { Establish } \\
\text { Shared Understanding }\end{array}$ & $-3.56^{*}$ & $-7.09--0.03$ & $\begin{array}{l}\text { Representing } \\
\text { and Formulating }\end{array}$ & 3.16 & $-0.02-6.35$ \\
Maintaining & & & & 3.44 & $-0.83-7.71$ \\
Communication & $-4.39^{* *}$ & $-7.18--1.61$ & Executing & 0.95 & $-4.13-6.02$ \\
Sharing Information & -1.85 & $-4.74-1.04$ & Monitoring & $46.29^{* * *}$ & $23.93-68.64$ \\
Negotiating & $-4.12^{*}$ & $-7.33--0.91$ & Planning & &
\end{tabular}

Table 5: CPS skills and productive decision change (group level)

\begin{tabular}{lcclcc}
\hline & \multicolumn{2}{c}{ Productive Decision Change } & \multicolumn{2}{c}{ Productive Decision Change } \\
CPS Skills (Social) & $\beta$ & $C I$ & CPS Skills (Cognitive) & $\beta$ & CI \\
\hline $\begin{array}{l}\text { Establish } \\
\text { Shared Understanding }\end{array}$ & -2.69 & $-6.08-0.71$ & $\begin{array}{l}\text { Representing } \\
\text { and Formulating }\end{array}$ & -1.49 & $-4.70-1.73$ \\
$\begin{array}{l}\text { Maintaining } \\
\text { Communication }\end{array}$ & -0.71 & $-3.38-1.96$ & Executing & $5.16^{*}$ & $0.86-9.45$ \\
Sharing Information & $2.88^{*}$ & $0.11-5.66$ & Monitoring & $-5.82^{*}$ & $-10.92--0.72$ \\
Negotiating & -1.55 & $-4.60-1.49$ & Planning & $23.10^{*}$ & $0.64-45.55$ \\
\hline
\end{tabular}

\subsection{Individual level CPS skills and outcomes}

Regarding the relationship between individual CPS skills and decision change, our results show that maintaining communication had a significant negative correlation with decision change. This indicates that individuals who exhibited more maintaining communication were less likely to change their answers. Since maintaining communication implies minimal engagement or off-topic discussion, individuals demonstrating this behavior might fail to gain 
effective information from group discussion and persist with their initial preference. The results also show a positive correlation between executing and individual decision change. This indicates that communicating actions positively correlates with the likelihood of individual shifting decision post discussion. This may further suggest action-oriented information may appeal to individuals to reconsider their initial positions.

Regarding the relationship between individual CPS skills and productive decision change, our results indicate that sharing information is significantly correlated with a positive decision change. This suggests that students who shared more information or established a shared understanding were more likely to have shifted to an improved decision after the group discussion. In other words, amongst those who gained useful insights from the group discussion to correct their decisions, they were more active contributors of shared information during group chats. Other social skills captured by the CPS framework were in slight negative correlations to making a productive decision change. The results also show no significant correlation between cognitive CPS skills and productive decision change. This indicates that productive changes in decisions are more a product of information exchange and interaction rather than individual CPS processes utilized to perform social regulation or group coordination.

\subsection{Group level CPS skills and outcomes}

Regarding group level CPS skills and the ratio of decision change in groups, our models indicate that establishing shared understanding, maintaining communication, and negotiating all have a significant negative correlation with group decision change whereas planning was positively correlated with group-level decision changes. The results could suggest that while individual decision changes are driven by suggesting and reporting actions for the group, grouplevel shifts in opinion rely more on planning. It is interesting to note that there has been inconsistent findings around negotiation. Some researchers found negotiation skill to be a positive attribute to successful collaboration[20], others suggest productive roles that drive successful collaboration in teams exhibited less negotiating behavior. Our results support the latter evidence. A possible explanation for this is that when there is a high degree of negotiation in the group, information might be too conflicting for members to be convinced and people might be more likely to succumb to the biases from initial preference.

Regarding group level CPS skills and the ratio of productive decision change in groups, our results show that sharing information had a significant positive correlation with productive group-level decision change. This could indicate that a productive shift in group opinion relies on unique information exchange from all group members. Additionally, executing and planning were also positively correlated with positive group-level decision change whereas monitoring had a negative correlation. This provides further nuances into the types of social regulatory activities that are most meaningful to groups. As the results imply, monitoring and checking in with team members updates in the instance of hidden-profile might be less beneficial to groups producing productive decision changes than suggesting actions and clarifying overall goals or proposing a problem-solving plan.
There are several limitations to the study. First of all, we did not account for the group level moderator variables that may influence the outcome. For instance, research have suggested group size and group composition may influence group interaction dynamics. Along that line, other individual attributes such as personality or demographic background such as gender and race were not controlled for in this study, yet may have inadvertently affect information sharing processes. Previous research found that gender. Furthermore, our analysis was unable to address questions regarding the importance of the revelation of key information. Although the experiment was set up such that positive outcome should be driven by shared key information, this analysis is insufficient to clarify the degree to which key information was shared in groups, nor to explain how unique information correlated to decision making outcomes. This is because CPS skills describe the actions in general and was less about the characteristics of information itself. However, as is stated in the introduction section, it is also an intentional angle that we chose differentiated from a concentration on information and task characteristics among many social psychology studies. We positioned through the lens of CPS skills so to understand and offer implications for CPS processes more broadly, beyond the hidden profile scenario. To this end, we plan to include a set of secondary codes that we assigned to each utterances to describe discourse action in greater details. For instance, the subcodes for sharing information include the scope of information being revealed (major versus minor reveal), feature types (positive versus negative information), redundancy(new versus already mentioned information).

\section{CONCLUSION AND FUTURE WORK}

Collaborative problem-solving skills as one of the key 21st-century competencies is essential for success in today's society. It has become increasingly evident that CPS skills play a crucial part in knowledge construction and decision making in teams across school and professional settings. Therefore, there is a need for more research efforts to expand our understanding of the roles of CPS competencies in team decision making.

Our contribution to this line of research is threefold. First, we used a multilevel analysis to reveal how different CPS skills affect decision-making outcomes differently across individuals and groups levels. Our results suggest that there is a slight difference in the way communication processes contribute to individuals and groups making effective judgments. For instance, establishing shared understanding only correlates with hindered decision change at the group level but not at the individual level. Moreover, we observe that the effect of the same CPS skills on outcomes was more pronounced at the group level than at the individual level. Our statistical models also show a more robust relationship with greater variance explained by CPS attributes in group models. This may suggest that CPS skills assessment might characterize social regulatory behavior that is associated with decision-making performance but existed only at the group level. Another potential explanation is that CPS assessment based on individual level contributions in isolation to team discourse might leave out interpersonal dynamics that could potentially affect decision-making outcomes. In our related work 
section, we mentioned research highlighting the interplay of intrapersonal and interpersonal communication during collaboration, and the rich information we can glean by taking into account the semantic relatedness of group discourse [11]. Our study substantiates the dynamic nature of collaborative interactions and their potential impact on decision making. For instance, we found that individuals who exhibited more information-sharing patterns were more likely to have improved their answers after group discussion. But we did not have direct evidence to show whether they also benefited from their teammates' information sharing. We suggest future research to investigate individual CPS behavior with respect to teammate's interactions to further unpack the interpersonal aspect of collaborative decision making.

Secondly, we provide some practical implications on CPS practices. We see an opportunity to provide explicit and actionable feedback to digitally-mediated decision making groups regarding specific CPS areas that they are better or worse at. The CPS framework is also useful for monitoring group behavior. For instance, overly high amounts of negotiating, can establish shared understanding and might signal information overload or states of confusion as these factors are predictive of failure to recognize the best alternative (i.e., persistent to initial preference). We also provide some directions in facilitating effective decision-making collaborations. Our findings suggest that assisting group planning and executing processes may be conducive to CPS outcomes. However, more replicated research and future studies in an empirical setting are necessary to validate these suggestions.

Lastly, we leverage cross-disciplinary knowledge to illuminate a novel way to study the hidden-profile paradigm. Combining insights from social psychology and learning analytics, our study not only presents a unique context for understanding collaboration problem solving skills and performance but also introduces an unconventional method to assess information sharing and processing in decision making literature. As a methodological contribution, we incorporated CPS framework in a hidden-profile task to uncover social and cognitive mechanisms associated with individual's and group's information sharing and processing. We found social and cognitive CPS skills together interpret information sharing and processing outcomes in a more meaningful way. Moreover, recent advances in learning analytics offer a prospective outlook of automated content analysis to perform CPS assessment more efficiently[39][20]. A recent study applied computational linguistics to identify sociocognitive roles and evaluated its relationship with CPS skills [11]. These emergent research opens up an unprecedented opportunity for potentially investigating CPS skills in the context of collaborative decision making at a larger scale.

In sum, our study integrates multiple perspectives that are at the core interests of the learning analytics community. Towards the current trend of development of LA research, it is imperative that diverse learning paradigms and settings should be spotlighted in addition to learning analytics approaches[30]. Although our study employs discourse analytics to unveil key characteristics of learner communication in collaborative learning at a broad level, we also aim to establish our understanding of learners' social and cognitive dimensions within a unique information distribution environment (i.e., Hidden-profile paradigm). As the field of learning analytics expands its taxonomy over various methodological, conceptual, and contextual domains, the pursuit of scalable and generalizable insights should also be coupled with fine-grained viewpoints that provide contextual depths and details. Moreover, along the effort for curating more diverse and equitable learning environment, learner differences should also be emphasized on research agendas[24].

In future studies, we plan to further disentangle the relationship of group process and individual perception of information in collaborative learning environment, with regards to learner characteristics including demographic attributes and knowledge background. We also aim to reflect and design feedback and intervention based on experimental findings to facilitate collaborative processes and knowledge exchange. Future collaborative analytics along this vein should gear towards the goal of informing design and artefacts that serve the needs of a diverse range of learning paradigms.

\section{ACKNOWLEDGMENTS}

This research was supported by The Andrew W. Mellon Foundation (1806-05902). The authors would like to thank the Next Generation Undergraduate Success Measurement Project team members for help with data collection.

\section{REFERENCES}

[1] Jessica Andrews-Todd and Carol M Forsyth. 2018. Exploring social and cognitive dimensions of collaborative problem solving in an open online simulation-based task. Comput. Human Behav. (Oct. 2018).

[2] S E Asch. 1951. Effects of group pressure upon the modification and distortion of judgments. In Groups, leadership and men; research in human relations, ( $p$, Harold Guetzkow (Ed.). Vol. 293. Carnegie Press, ix, Oxford, England, 177-190.

[3] Solomon E Asch. 1955. Opinions and Social Pressure. Sci. Am. 193, 5 (1955), 31-35.

[4] Suzanne T Bell. 2007. Deep-level composition variables as predictors of team performance: a meta-analysis. Journal of applied psychology 92, 3 (2007), 595.

[5] Z. Cai, Brendan R. Eagan, N. Dowell, J. Pennebaker, A. Graesser, and D. W. Shaffer. 2017. Epistemic Network Analysis and Topic Modeling for Chat Data from Collaborative Learning Environment. In EDM.

[6] Esther Care, Claire Scoular, and Patrick Griffin. 2016. Assessment of collaborative problem solving in education environments. Applied Measurement in Education 29, 4 (Oct. 2016), 250-264

[7] Vanessa Dayeh and Ben W Morrison. 2020. The Effect of Perceived Competence and Competitive Environment on Team Decision-Making in the Hidden-Profile Paradigm. Group Decision and Negotiation (2020), 1-25.

[8] Bram De Wever, Hilde Van Keer, Tammy Schellens, and Martin Valcke. 2007. Applying multilevel modelling to content analysis data: Methodological issues in the study of role assignment in asynchronous discussion groups. Learning and Instruction 17, 4 (Aug. 2007), 436-447.

[9] Nia Dowell, Yiwen Lin, Andrew Godfrey, and Christopher Brooks. 2019. Promoting inclusivity through time-dynamic discourse analysis in digitally-mediated collaborative learning. In International Conference on Artificial Intelligence in Education. Springer, 207-219.

[10] Nia MM Dowell, Christopher Brooks, Vitomir Kovanović, Srećko Joksimović, and Dragan Gašević. 2017. The changing patterns of MOOC discourse. In Proceedings of the Fourth (2017) ACM Conference on Learning@ Scale. 283-286.

[11] N M Dowell, Yiwen Lin, Andrew Godfrey, and Christopher Brooks. 2020. Exploring the relationship between emergent sociocognitive roles, collaborative problem- solving skills and outcomes: A Group Communication Analysis. Fournal of Learning Analytics 7, 1 (April 2020), 38-57.

[12] Nia MM Dowell, Tristan M Nixon, and Arthur C Graesser. 2019. Group communication analysis: A computational linguistics approach for detecting sociocognitive roles in multiparty interactions. Behavior research methods 51, 3 (2019), 1007-1041.

[13] Nia M.M. Dowell and Oleksandra Poquet. 2021. SCIP: Combining group communication and interpersonal positioning to identify emergent roles in scaled digital environments. Computers in Human Behavior 119 (2021), 106709. https://doi.org/10.1016/j.chb.2021.106709

[14] Nia Marcia Maria Dowell and Arthur C Graesser. 2014. Modeling learners' cognitive, affective, and social processes through language and discourse. Fournal of Learning Analytics 1, 3 (2014), 183-186. 
[15] Stephen M Fiore, Arthur Graesser, and Samuel Greiff. 2018. Collaborative problemsolving education for the twenty-first-century workforce. Nature Human Behaviour 2, 6 (June 2018), 367.

[16] Arthur Graesser, Peter W Foltz, Yigal Rosen, David Williamson Shaffer, Carol Forsyth, and Mae-Lynn Germany. 2018. Challenges of Assessing Collaborative Problem Solving. In Assessment and Teaching of 21st Century Skills: Research and Applications, Esther Care, Patrick Griffin, and Mark Wilson (Eds.). Springer International Publishing, Cham, 75-91.

[17] Arthur C Graesser, Stephen M Fiore, Samuel Greiff, Jessica Andrews-Todd, Peter W Foltz, and Friedrich W Hesse. 2018. Advancing the Science of Collaborative Problem Solving. Psychol. Sci. Public Interest 19, 2 (Nov. 2018), 59-92.

[18] Patrick Griffin, Barry McGaw, and Esther Care (Eds.). 2012. Assessment and Teaching of 21st Century Skills. Springer, Dordrecht.

[19] Deborah H Gruenfeld, Elizabeth A Mannix, Katherine Y Williams, and Margaret A Neale. 1996. Group composition and decision making: How member familiarity and information distribution affect process and performance. Organizational behavior and human decision processes 67, 1 (1996), 1-15.

[20] Jiangang Hao, Lei Liu, Alina A von Davier, Nathan Lederer, Diego Zapata-Rivera, Peter Jakl, and Michael Bakkenson. 2017. EPCAL: ETS Platform for Collaborative Assessment and Learning: EPCAL. ETS Research Report Series 2017, 1 (Dec. 2017), $1-14$.

[21] Jeroen Janssen, Gijsbert Erkens, Paul A Kirschner, and Gellof Kanselaar. 2011 Multilevel Analysis in CSCL Research. In Analyzing Interactions in CSCL: Methods, Approaches and Issues, Sadhana Puntambekar, Gijsbert Erkens, and Cindy HmeloSilver (Eds.). Springer US, Boston, MA, 187-205

[22] D Marc Kilgour and Colin Eden. 2010. Handbook of group decision and negotiation. Vol. 4. Springer Science \& Business Media.

[23] J R Landis and G G Koch. 1977. The measurement of observer agreement for categorical data. Biometrics 33, 1 (March 1977), 159-174.

[24] Yiwen Lin, Nia Dowell, Andrew Godfrey, Heeryung Choi, and Christopher Brooks. 2019. Modeling gender dynamics in intra and interpersonal interactions during online collaborative learning. In Proceedings of the 9th international conference on learning analytics \& knowledge. 431-435.

[25] Lei Liu, Jiangang Hao, Alina A von Davier, Patrick Kyllonen, and Juan-Diego Zapata-Rivera. 2016. A tough nut to crack: Measuring collaborative problem solving. In Handbook of research on technology tools for real-world skill development. IGI Global, 344-359.

[26] Daniel J Mallinson and Peter K Hatemi. 2018. The effects of information and social conformity on opinion change. PloS one 13, 5 (2018), e0196600.

[27] John Mathieu, M Travis Maynard, Tammy Rapp, and Lucy Gilson. 2008. Team effectiveness 1997-2007: A review of recent advancements and a glimpse into the future. Journal of management 34, 3 (2008), 410-476.

[28] Shinichi Nakagawa and Holger Schielzeth. 2013. A general and simple method for obtaining R2 from generalized linear mixed-effects models. Methods Ecol. Evol. 4, 2 (Feb. 2013), 133-142.

[29] OECD. 2017. PISA 2015 assessment and analytical framework: Science, reading, mathematic, financial literacy and collaborative problem solving. Technical Report. Organisation for Economic Co-operation and Development.

[30] Alejandro Peña-Ayala. 2018. Learning analytics: A glance of evolution, status, and trends according to a proposed taxonomy. Wiley Interdisciplinary Reviews Data Mining and Knowledge Discovery 8, 3 (2018), e1243.

[31] José Pinheiro and Douglas Bates. 2006. Mixed-effects models in S and S-PLUS. Springer Science \& Business Media.

[32] J Pinheiro, Douglas Bates, Saikat DebRoy, Deepayan Sarkar, Siem Heisterkamp, and Bert sigma). 2016. nlme: Linear and nonlinear mixed effects models.

[33] Torsten Reimer, Andrea Reimer, and Uwe Czienskowski. 2010. Decision-making groups attenuate the discussion bias in favor of shared information: A metaanalysis. Communication Monographs 77, 1 (2010), 121-142.

[34] Sylvia G Roch and Roya Ayman. 2005. Group decision making and perceived decision success: The role of communication medium. Group dynamics: theory, research, and practice 9, 1 (2005), 15 .

[35] Jeremy Roschelle and Stephanie D Teasley. 1995. The construction of shared knowledge in collaborative problem solving. In Computer supported collaborative learning. Springer, 69-97.

[36] Dominique S Rychen and Laura H Salganik. 2003. Key Competencies for a Successful Life and Well-Functioning Society. Hogrefe Publishing.

[37] Stefan Schulz-Hardt and Andreas Mojzisch. 2012. How to achieve synergy in group decision making: Lessons to be learned from the hidden profile paradigm. European Review of Social Psychology 23, 1 (2012), 305-343.

[38] Claire Scoular and Esther Care. 2019. Monitoring patterns of social and cognitive student behaviors in online collaborative problem solving assessments. Comput Human Behav. (Jan. 2019).

[39] Claire Scoular, Esther Care, and Friedrich W Hesse. 2017. Designs for operationalizing collaborative problem solving for automated assessment. fournal of Educational Measurement 54, 1 (2017), 12-35.

[40] Michael Smilowitz, D Chad Compton, and Lyle Flint. 1988. The effects of computer mediated communication on an individual's judgment: A study based on the methods of Asch's social influence experiment. Computers in Human Behavior 4
4 (1988), 311-321.

[41] Serena G Sohrab, Mary J Waller, and Seth Kaplan. 2015. Exploring the hiddenprofile paradigm: A literature review and analysis. Small Group Research 46, 5 (2015), 489-535.

[42] Garold Stasser and William Titus. 1985. Pooling of unshared information in group decision making: Biased information sampling during discussion. f. Pers. Soc. Psychol. 48, 6 (1985), 1467-1478.

[43] Garold Stasser and William Titus. 2003. Hidden Profiles: A Brief History. Psychol. Inq. 14, 3-4 (Oct. 2003), 304-313.

[44] Garold Stasser and William Titus. 2003. Hidden profiles: A brief history. Psychological Inquiry 14, 3-4 (2003), 304-313.

[45] Yla Tausczik and Xiaoyun Huang. 2019. The Impact of Group Size on the Discovery of Hidden Profiles in Online Discussion Groups. ACM Transactions on Social Computing 2, 3 (2019), 1-25.

[46] J Lukas Thürmer, Frank Wieber, Thomas Schultze, and Stefan Schulz-Hardt. 2018. Hidden profile discussion coding tracing synergy in group decisions. (2018).

[47] Alieke M van Dijk, Tessa H S Eysink, and Ton de Jong. 2020. Supporting Cooperative Dialogue in Heterogeneous Groups in Elementary Education. Small Group Research 51, 4 (Aug. 2020), 464-491.

[48] Ethlyn A Williams and Stephanie L Castro. 2010. The effects of teamwork on individual learning and perceptions of team performance. Team Performance Management: An International fournal (2010).

[49] Gwen M Wittenbaum, Andrea B Hollingshead, and Isabel C Botero. 2004. From cooperative to motivated information sharing in groups: Moving beyond the hidden profile paradigm. Communication Monographs 71, 3 (2004), 286-310.

[50] Jianlin Yuan, Yue Xiao, and Hongyun Liu. 2019. Assessment of Collaborative Problem Solving Based on Process Stream Data: A New Paradigm for Extracting Indicators and Modeling Dyad Data. Front. Psychol. 10 (Feb. 2019), 369.

[51] Diego Zapata-Rivera, Tanner Jackson, Lei Liu, Maria Bertling, Margaret Vezzu, and Irvin R Katz. 2014. Assessing Science Inquiry Skills Using Trialogues. In Intelligent Tutoring Systems. Springer International Publishing, 625-626. 